\title{
Oftalmomiasis por Oestrus ovis en un área urbana al sur del Perú
}

'Universidad Nacional de San Agustín de Arequipa. Arequipa,

Recibido: 8 de enero de 2019 Aceptado: 9 de mayo de 2019

Correspondencia a: Oscar Moreno-Loaiza oscarml5@hotmail.com

\section{Oestrus ovis ophthalmomyiasis at an urban area in Southern Peru}

Ophthalmomyiasis is the ocular infestation by dipterous larvae. It has been described in patients from rural areas, poor hygiene, or contact with animals. However, there are sporadic reports of this infestation in urban areas. We present the case of a young man without risk factors, from an urban area in southern Peru, in whom we found 10 larvae at ocular conjunctiva. The larvae were identified as Oestrus ovis, a fly that usually infests sheep and goats as part of their life cycle. We made a brief review of the case, highlighting the importance of adequately identifying the species that cause myiasis as well as its life cycle.

Keywords: Myiasis; larva; eye foreign body; zoonoses.

Palabras clave: Miasis; larva; cuerpo extraño ocular; zoonosis.

\section{Introducción}

L a miasis es una parasitosis en la que larvas de dípteros infestan tejidos sanos o lesionados de un hospedero ${ }^{1}$. Esta condición ha sido descrita en diversos países del mundo. Sin embargo, es más frecuente en países tropicales debido a condiciones ambientales favorables a su desarrollo y la presencia endémica de especies de dípteros que requieren infestar mamíferos $u$ otros animales como parte de su ciclo de vida ${ }^{2}$.

La afección ocular por larvas u oftalmomiasis es frecuente en animales, pero de rara ocurrencia en seres humanos. Los factores de riesgo más importantes para su desarrollo son condiciones de higiene deficientes, situación de abandono o indigencia, y contacto cercano con animales o granjas ${ }^{3-5}$. A pesar de ello, existen reportes esporádicos de oftalmomiasis en personas sin factores de riesgo o en áreas urbanas ${ }^{6}$. En Latinoamérica, existen escasos casos descritos de esta afección, siendo la mayoría de ellos en áreas tropicales o de selva, y con factores de riesgo marcados para este tipo de infección ${ }^{7-10}$.

La oftalmomiasis presenta tres formas clínicas: externa, intraocular y orbitaria ${ }^{11}$. Distintas especies y condiciones pueden favorecer el desarrollo de las mis$\operatorname{mas}^{7-9,12}$. Su gravedad depende de la profundidad del compromiso de tejido, la forma de contagio y el estado inmune del paciente.

Se presenta el caso de un paciente con infestación por larvas en la superficie ocular (oftalmomiasis externa). Debe considerarse la posibilidad diagnóstica de miasis en pacientes sin factores de riesgo, y enfatizar la importancia de una adecuada identificación de la especie causante de la infestación para poder realizar un adecuado tratamiento y manejo posterior.

\section{Caso Clínico}

Varón de 26 años, procedente de un área urbana al sur de Perú, que acudió al servicio de urgencias refiriendo que cinco horas atrás, mientras se encontraba caminando en un parque, presentó en forma súbita una sensación de cuerpo extraño, lagrimeo y enrojecimiento en el ojo izquierdo. Tenía buenas condiciones de higiene, sin antecedentes médicos de importancia, ni viajes a regiones rurales en los últimos 6 meses. Su ocupación era chef, contaba con un ingreso mediano, y exámenes médicos periódicos. No refirió consumo de alcohol en forma regular o abuso de drogas.

El examen físico general era normal. La agudeza visual era 20/20 sin correctores en ambos ojos, con reflejos fotomotores conservados. No evidenciamos proptosis, ni signos inflamatorios en los párpados. Al ser evaluado en la lámpara de hendidura se encontró una leve hiperemia de la conjuntiva bulbar, sin lesiones corneales y la presencia de 10 larvas móviles de 1 a 1,5 mm, localizadas en los fondos de saco conjuntivales superior e inferior. La cámara anterior no presentaba signos inflamatorios. El fondo de ojo no tenía alteraciones aparentes.

Se retiraron todas las larvas visibles en forma manual, con un hisopo y bajo anestesia tópica. El paciente fue manejado ambulatoriamente, y se le indicó colirio de ciprofloxacino $0,3 \%$ con dexametasona $0,1 \%$ cada $4 \mathrm{~h}$, durante 7 días. 
En el control al primer día no se encontró ninguna larva. Sin embargo, en el control del tercer día, el paciente refirió haber eliminado una larva de $10 \mathrm{~mm}$ por la nariz. Fue evaluado por especialistas de otorrinolaringología, quienes no encontraron signos de necrosis o inflamación en la cavidad nasal. Se realizó una tomografía de senos paranasales y endoscopía nasal, en la que no se evidenció presencia de larvas.

En tanto, las larvas extraídas fueron enviadas al Laboratorio de Parasitología de la Universidad Nacional San Agustín de Arequipa (UNSA), Perú. En la observación directa al microscopio se observó una larva segmentada con múltiples espinas en la superficie ventral y un par de ganchos en el polo anterior (Figuras 1 y 2). En colaboración con un especialista en entomología de la UNSA y confirmación en el Instituto Nacional de Salud (INS), Lima-Perú, se llegó a identificar la larva como Oestrus ovis.

\section{Discusión}

Oestrus ovis, también conocida como mosca de las ovejas, afecta principalmente ovejas y cabras, como parte de su ciclo vital. Esta mosca larvípara, coloca las larvas en ojos u hocicos de los animales, desde donde migran a los senos paranasales, alimentándose de secreciones mucosas durante semanas o meses ${ }^{5}$. Posteriormente, son eliminadas junto con las secreciones nasales para formar una pupa en el suelo ${ }^{3}$.

Oestrus ovis deposita rápidamente sus larvas en el hospedero, sin necesidad de interrumpir su vuelo ${ }^{6,13}$, lo que podría explicar que el paciente no haya visto a la mosca.

Existen casos descritos de oftalmomiasis por Oestrus ovis en granjeros y personas indigentes de áreas rurales, que actúan como hospederos accidentales de este parásito $^{3,5,13}$. En el caso presentado, el paciente no refirió contacto con animales ni realizó visitas a zonas rurales. La infestación ocurrió posiblemente durante su estadía en un parque de la ciudad. A pesar de haber ocurrido en un área urbana, hay que destacar que en la periferia de la ciudad existe actividad ganadera, desde donde podría haber llegado esta especie de mosca. Esta hipótesis demostraría una alta movilidad de esta especie, y que no solo se encontraría confinada en lugares en que su hospedero natural (ovejas y cabras) se encuentra.

Esta situación es similar a una serie de casos de oftalmomiasis en Turquía, donde la mayoría se presentaron en áreas urbanas y en personas sin contacto con animales ${ }^{4}$. Otros autores, han señalado la posibilidad de una contaminación en jardines y parques de áreas urbanas, fertilizados con material proveniente de granjas de ovejas y cabras, donde podrían encontrarse las pupas de las moscas ${ }^{14}$.

En el Perú se han reportado escasos casos de oftalmo- miasis en pacientes provenientes de regiones rurales ${ }^{5} \mathrm{O}$ con antecedente de alcoholismo y contacto con animales ${ }^{15}$. Siendo este un diagnóstico infrecuente, orientamos el objetivo del tratamiento en evitar mayor daño a los tejidos y prevenir la aparición de una conjuntivitis bacteriana secundaria.

En este caso, poco convencional en la práctica de oftalmología, debe resaltarse la importancia de identificar adecuadamente la especie causante de la enfermedad. En algunos casos se ha recomendado conservar las larvas vivas y dejarlas madurar a su estado adulto (mosca) para poder identificarlas adecuadamente ${ }^{16}$. Sin embargo, esto puede ser poco práctico en el escenario clínico común y en ausencia de peritos en entomología, por lo que una aproximación inicial adecuada es extraer las larvas

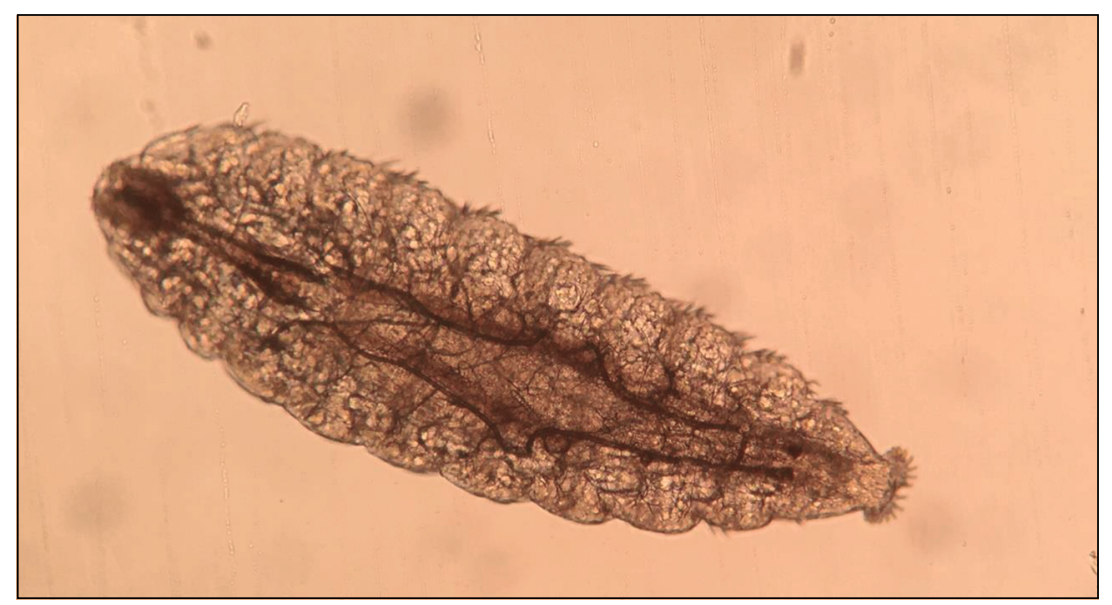

Figura 1. Larva extraída del fondo de saco conjuntival del ojo izquierdo del paciente (visión bajo microscopio, aumento 100X).

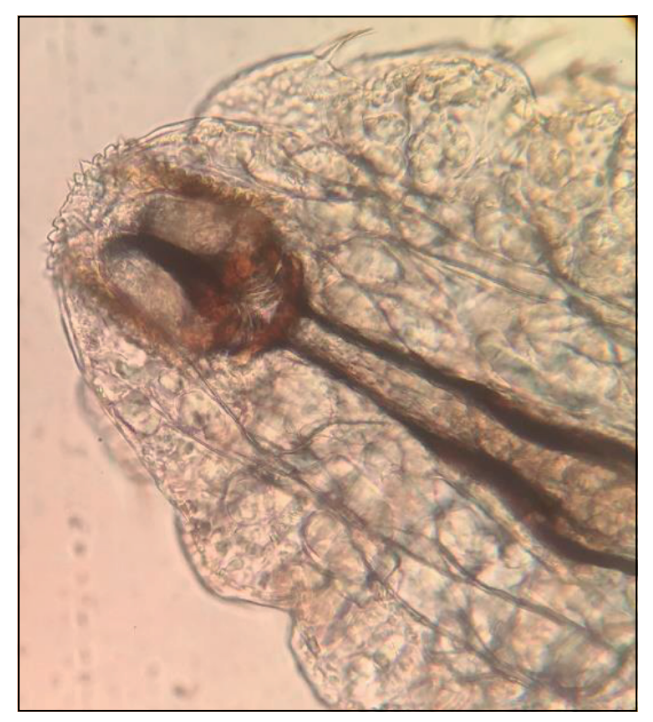

Figura 2. Polo anterior de Oestrus ovis con detalles característicos de la especie (visión bajo microscopio, aumento 400X). 
evitando dañar los extremos anterior y posterior de la misma para permitir su identificación en un laboratorio especializado.

En conclusión, aunque poco frecuente, Oestrus ovis puede causar oftalmomiasis en pacientes sin factores de riesgo. El conocimiento de la especie infectante, así como su ciclo de vida, es necesario para planear un tratamiento y seguimiento adecuados en un paciente con esta parasitosis.

Agradecimientos: A Nancy Ruelas del Departamento de Microbiología y Patología de la Universidad Nacional de San Agustín de Arequipa y a María Beltrán del Instituto Nacional de Salud, por su colaboración en la identificación de la especie del parásito.

\section{Resumen}

La oftalmomiasis es la infestación ocular por larvas de dípteros. Ha sido descrita en pacientes provenientes de áreas rurales, higiene deficiente o por contacto con animales. Sin embargo, existen reportes esporádicos de esta infestación en áreas urbanas. Presentamos el caso de un varón joven, sin factores de riesgo, proveniente de un área urbana al sur del Perú, que presentó 10 larvas en la conjuntiva ocular. Las larvas fueron identificadas como Oestrus ovis, una mosca que suele infestar ovejas y cabras como parte de su ciclo de vida. Realizamos una breve revisión del tema, resaltando la importancia de identificar adecuadamente la especie causante de la miasis, así como su ciclo de vida.

\section{Referencias bibliográficas}

1.- $\quad$ Singh A, Singh Z. Incidence of myiasis among humans-a review. Parasitol Res 2015; 114: 3183-99. doi:10.1007/s00436-015-4620-y.

2.- Solomon M, Lachish T, Schwartz E. Cutaneous myiasis. Curr Infect Dis Rep 2016; 18: 28. doi:10.1007/s11908-016-0537-6.

3.- Sucilathangam G, Meenakshisundaram A, Hariramasubramanian S, Anandhi D, Palaniappan N, Anna T. External ophthalmomyiasis which was caused by sheep botfly (Oestrus ovis) larva: a report of 10 cases. J Clin Diagn Res 2013; 7(3): 539-542. doi:10.7860/JCDR/2013/4749.2817.

4.- Özyol P, Özyol E, Sankur F. External ophthalmomyiasis: a case series and review of ophthalmomyiasis in Turkey. Int Ophthalmol 2016; 36: 887-91. doi:10.1007/s10792-0160204-9.

5.- Beltrán F M, Torres V G, Segami S H, Náquira V C. Miasis ocular por Oestrus ovis. Rev Peru Med Exp Salud Publica 2006; 23: 70-2. doi:http://dx.doi.org/10.17843/ rpmesp.2006.231.1038.
6.- Nisrine L, Taoufik A, Zaoui K, et al. External ophthalmomyiasis: a case report from urban area. Indian J Med Spec 2017; 8:164-166. doi:10.1016/J.INJMS.2017.08.004.

7.- Domínguez Enríquez J, Cueva Rosillo J, Cusco Cuzco C, Rodríguez-Hidalgo R, Calvopiña M. Miasis orbital severa causada por Cochliomyia hominivorax en la región andina de Ecuador. Rev Mex Oftalmol 2016; 90: 43-47. doi:10.1016/J.MEXOFT.2015.05.008

8.- Von Faber Bison S H D, De Campos Machado M A, Ferrari Silva J A, García E A, Ramírez Dittrich M A. Lacrimal sac topography myiais. Rev Bras Oftalmol 2016; 75: 67-9. doi:10.5935/0034-7280.20160016.

9.- Saraiva V da S, Amaro M H, Belfort R Jr, Burnier M N Jr. A case of anterior internal ophthalmomyiasis: case report. Arq Bras Oftalmol 2006; 69: 741-3. doi:10.1590/S0004 27492006000500023.

10.- Estrada-Amaya R, López M. Oftalmomiasis. Reporte de caso. Rev Sanid Milit Mex 2016; 70: 389-92.

11.- Panadero-Fontán R, Otranto D. Arthropods affecting the human eye. Vet Parasitol 2015;
208: 84-93. doi:10.1016/j.vetpar.2014.12.022.

12.- Baliga M J, Davis P, Rai P, Rajasekhar V. Orbital myiasis: a case report. Int J Oral Maxillofac Surg 2001; 30: 83-4. doi:10.1054/ IJOM.2000.0007.

13.- Gregory A R, Schatz S, Laubach H. Ophthalmomyiasis caused by the sheep bot fly Oestrus ovis in Northern Iraq. Optom Vis Sci 2004; 81: 586-90. doi:10.1097/01. opx.0000141793.10845.64.

14.- D'assumpcao C, Bugas A, Heidari A, Sofinski S, Mcpheeters R. 194 ophthalmomyiasis caused by sheep bot fly (Oestrus ovis) in Central Valley, California, United States. J Investig Med 2018; 66: A146-7. doi:10.1136/jim-2017000663.194.

15.- Hoyer P, Williams R R, López M, Cabada $\mathrm{M}$ M. Human nasal myiasis caused by Oestrus ovis in the Highlands of Cusco, Peru: report of a case and review of the literature. Case Rep Infect Dis 2016; 2016: 2456735. doi:10.1155/2016/2456735.

16.- Tabbara K F, El-Asrar A M A, Khraillah M, editors. Ocular Infections. 1st ed. Springer Berlin Heidelberg; 2014. 